\title{
A Classification of Decision Automation and Delegation in Digital Investment Management Systems
}

\author{
Alexander Rühr \\ LMU Munich \\ ruehr@bwl.lmu.de
}

\author{
David Streich \\ LMU Munich \\ streich@bwl.lmu.de
}

\author{
Benedikt Berger \\ LMU Munich \\ benedikt.berger@bwl.lmu.de
}

\author{
Thomas Hess \\ LMU Munich \\ thess@bwl.lmu.de
}

\begin{abstract}
Digital investment management systems, commonly known as robo-advisors, provide new alternatives to traditional human services, offering competitive investment returns at lower cost and customer effort. However, users must give up control over their investments and rely on automated decision-making. Because humans display aversion to high levels of automation and delegation, it is important to understand the interplay of these two aspects. This study proposes a taxonomy of digital investment management systems based on their levels of decision automation and delegation along the investment management process. We find that the degree of automation depends on the frequency and urgency of decisions as well as the accuracy of algorithms. Notably, most providers only invest in a subset of funds pre-selected by humans, potentially limiting efficiency gains. Based on our taxonomy, we identify archetypical system designs, which facilitate further research on perception and adoption of digital investment management systems.
\end{abstract}

\section{Introduction}

Advancements in technological capabilities have facilitated the digitalization of a growing number of services. Most recently, the financial services industry has been subject to the emergence of digital solutions, including digital transaction, lending, trading and advisory services $[1,2]$. In investment management, private investors delegate an increasing number of decisions to digital systems, commonly referred to as robo-advisors [3, 4]. This phenomenon is driven by efficiency gains from increasing automation and delegation. Previous research has shown that human investment management does not provide superior returns compared to fully automated investment management [5]. At the same time, robo-advisors have been shown to improve retail investors' performance while saving customers time and mental effort [6-10]. However, robo-advisors have not yet been able to capture substantial market shares [3]. This raises the question of which factors might inhibit the use of digital investment management. Information systems (IS) research yields two potential explanations for this question. Users have been shown to display an aversion to algorithmic decision systems [11] as well as to the perceived loss of control associated with excessive delegation of decision authority [12]. It therefore stands to reason that a trade-off between benefits and costs of increasing decision automation as well as delegation is emerging in the design of digital investment management systems. Automation refers to the way in which decision-making is conducted by a support system, whereas delegation refers to the extent of user involvement in decisions.

To gain a better understanding of the service designs in digital investment management available to potential users, we propose the following research question: To what extent do existing digital investment management systems automate and involve customers in the investment management process? Our analysis proceeds in two steps. First, we propose a taxonomy of decision automation and delegation inherent in current systems in an iterative procedure based on the methodology proposed by Nickerson et al. [13]. Second, we draw upon the findings and concepts developed in the taxonomy to derive archetypical patterns of decision automation and delegation. Based on the findings from these two steps, we identify critical combinations of automation and delegation and pose new questions for system design.

Thus, we contribute to IS literature by integrating decision automation and delegation considerations in the context of digital investment management. Studies in this particular domain of IS research have solidified knowledge of decision delegation to autonomous algorithmic systems. We extend this knowledge to account for the complexity of this phenomenon resulting from the interaction of automation and delegation in the sensitive context of personal finance.

The remainder of this paper is structured as follows: In section 2, we introduce investment management as a service and describe the functionality of digital investment management systems before elaborating on 
related studies which further put our study into context. Following an introduction to the methodological approach, we present and discuss the iteration steps leading to our taxonomy in section 3. We derive and discuss archetypes of digital investment management systems based on our taxonomy in section 4. Section 5 concludes, outlines the contribution of this paper and suggests avenues for further research.

\section{Background}

\section{1. (Digital) investment management}

Research in personal finance has documented investors' lack of financial literacy, which is reflected in suboptimal investment behavior [14]. Professional investment management has been discussed regarding its potential to compensate financial illiteracy [15] and alleviate behavioral biases [16].

For the purpose of this study, we view investment management as a means to achieve an individual's financial goals over a pre-specified time horizon. To identify investor risk profiles, traditional advisors initiate investor contact and engage in face-to-face dialogues (see Figure 1). Suitable investment products are identified and allocated weights to form a portfolio concept consistent with this risk profile, which is then presented to the investor. In holistic services the proposed portfolio is implemented upon agreement and maintained to accord with the risk profile over time [17].

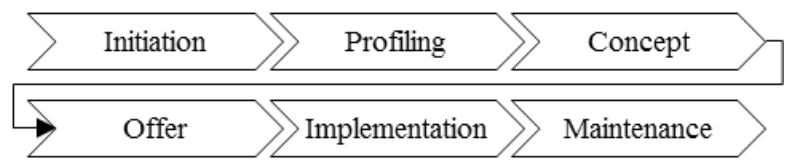

Figure 1: Investment management process from Nüesch et al. [17]

Digital investment management systems are browser or mobile applications capable of digitalizing the entire investment management process [3, 18]. Based on an online questionnaire, these systems create a risk profile, which is either depicted in categories such as 'conservative' and 'speculative' or as a quantitative risk measure such as the desired value at risk (VaR). In analogy to a traditional investment advisor, digital services pre-select investment products considered suitable for portfolio allocation. Due to the low-cost diversification strategy applied by most providers, portfolios tend to consist mostly of exchange-traded funds (ETFs). ETFs can replicate price developments of underlying asset classes ranging from real estate and commodities to stocks and bonds of all kinds.
Digital investment management systems use algorithms to assign portfolio weights to the preselected products based on user risk profiles. As the portfolio composition is affected by changes in market prices, the underlying risk of the portfolio can vary even in the absence of trades. Thus, portfolio risk is constantly monitored and corresponding trades are triggered to realign desired and actual portfolio risk.

Scholars have studied the design of robo-advisors [3, $4,19]$, as well as their ability to improve diversification and performance [20], eliminate behavioral biases [7] and assess risk preferences [21]. In the legal domain, potential conflicts of interest and liability issues have been discussed [22, 23]. However, the degree to which digital services take control of and automate investment management decisions has not been investigated.

\subsection{Automation and delegation}

Algorithms are shown to make decisions resulting in superior outcomes compared to those made by humans in many fields of application [6]. In the particular case of digital investment management systems, adoption of algorithms is associated with significantly improved market-adjusted investment returns [7]. This superiority is confirmed in an extensive meta-analysis of studies comparing algorithmic and human decision-making in psychological and medical applications [8].

Superior performance is countered by a general skepticism towards algorithmic decision systems. Previous studies have concluded that users tend to prefer human decision-making to algorithmic conduct [11, 24]. In human resources application, participants justified their aversion by claiming that algorithmic approaches were either less professional and less flexible [25] or unable to apply intuitive judgement in the same way a human would [26]. When delegating a medical decision to a human, patients reported a stronger decrease in perceived responsibility than for algorithms. This is in line with higher levels of trust in human decisions [27, 28]. Even when individuals witness algorithmic outperformance, they prefer the human delegate [11].

Studies on system adoption address the reluctance of users to relinquish control over a decision. The degree of delegation is negatively associated with perceived user control [9, 29], which evokes discomfort and anxiety in users [30]. In addition, highly autonomous systems are perceived as more risky and complex [29]. However, users are willing to accept at least partial loss of control in order to benefit from the efficiency gains from increased delegation [9], resulting from decreased mental effort and time saving [10]. 
While there have been studies separately investigating the impact of decision automation and delegation on system adoption, to date there has been no study integrating these two aspects. This study develops the basis for a holistic investigation of system adoption by evaluating investment management process steps regarding both aspects. As illustrated in Table 1, decision-making within a single process step can be conducted applying seven different combinations of automation and delegation.

\section{Table 1: Integration of automation and delegation}

\begin{tabular}{|c|c|c|c|c|}
\hline & & \multicolumn{3}{|c|}{ Level of delegation } \\
\hline & & None & Partial & Full \\
\hline \multirow{3}{*}{ 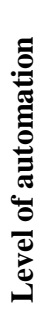 } & Human & $\begin{array}{c}\text { Self- } \\
\text { management }\end{array}$ & $\begin{array}{l}\text { Human } \\
\text { advice }\end{array}$ & $\begin{array}{l}\text { Delegation to } \\
\text { human }\end{array}$ \\
\hline & Hybrid & & $\begin{array}{l}\text { Hybrid } \\
\text { advice }\end{array}$ & $\begin{array}{l}\text { Delegation to } \\
\text { hybrid system }\end{array}$ \\
\hline & $\begin{array}{l}\text { Algo- } \\
\text { rithm }\end{array}$ & & $\begin{array}{l}\text { Algorithmic } \\
\text { advice }\end{array}$ & $\begin{array}{l}\text { Delegation to } \\
\text { algorithm }\end{array}$ \\
\hline
\end{tabular}

For the level of automation, we distinguish between human conduct (no automation), algorithmic conduct (full automation) and hybrid forms on the advisor's part [31]. For the level of delegation, we distinguish between no delegation (self-management irrespective of automation level), full delegation (no user involvement) and partial delegation. The threefold differentiation balances representativeness and conciseness and lends from an aggregation [31] of the automation classification suggested by Parasuraman et al. [32]. It is important to note that we define human conduct as (partially or fully) delegated human decision-making as part of service provision (i.e. human advisory employees), rather than user involvement. Thus, when referring to human conduct, we imply that decisions are made by a human advisor rather than the user. Decision involvement by the (human) user is only reflected in the level of delegation and not in the level of automation.

In allowing automation and delegation to interact, we combine insights from studies concerning algorithm aversion [11, 24] and user preference for involvement [12]. The former is concerned with the consideration between human and algorithmic conduct given a distinct level of delegation and is represented as variation within columns of Table 1. The latter investigates various levels of delegation given a distinct level of automation and is represented as variation within rows of Table 1.

\section{Taxonomy of delegation and automation}

Taxonomies have contributed to the comprehension of complex relationships and technological developments in the IS domain, supporting theory building in evolving areas of research $[1,33,34]$. To ensure scientific rigor in the development of classifications, Nickerson et al. [13] have proposed an iterative procedure around a pre-defined metacharacteristic reflecting the purpose of the analysis. The adjustments of the taxonomy in the various iterations aim to satisfy the specified universal ending conditions. Following this procedure, a taxonomy consists of $n$ dimensions $D_{i}(i=1, \ldots, n)$ and $k_{i}\left(k_{i} \geq 2\right)$ characteristics $C_{i j}$ $\left(j=1, \ldots, k_{j}\right)$ :

$$
T=\left\{D_{i}, i=1, \ldots, n \mid D_{i}=\left\{C_{i j}, j=1, \ldots, k_{i} ; k_{i} \geq 2\right\}\right\}
$$

\subsection{Taxonomy development}

To apply existing knowledge in our area of research, we adopt a conceptual-to-empirical approach to taxonomy development [13]. Therefore, we first conceptualize dimensions and respective characteristics from existing theory, which are calibrated based on collected data in multiple iterations. Following each iteration, the preliminary classification is reviewed with respect to the subjective and objective ending conditions determining the completion of the taxonomy. The metacharacteristic guiding the analyzed dimensions and characteristics in this study is the decision-making in digital investment management systems.

The first iteration is split into two parts, which are concerned with developing initial dimensions $(l a)$ and characteristics $(1 b)$ respectively. This initial taxonomy is then refined in iterations 2 and 3, after which all ending conditions are met.

Iteration $1 a$ - This iteration defines the investment process steps that function as dimensions of our taxonomy. Therefore, we derived an end-to-end process logic consisting of six core and 22 sub-process steps which range from initial customer contact to the operationalization of contracts [17]. This comprehensive initial process was verified using the official financial planning process outlined by the Financial Planning Standards Board (FPSB) [35], an incorporation of financial planning entities. In addition, we cross-checked the process using studies concerned with financial advice in general [36], as well as studies specifically examining digital investment management $[3,18]$. To accord with our meta-characteristic, nine out of 22 sub-process steps were excluded as they did not contain relevant decision-making processes. In addition, two steps regarding the identification of further advisory potential and the negotiation of the advisory agreement 
were dropped since they did not apply to digital investment management systems [18]. The remaining eleven steps were tested against observations from a subset of previously investigated systems [3]. In line with the process aggregation of the FPSB, all five subprocess steps with the aim of collecting client information were consolidated with the two steps directly utilizing this information to form the dimension $D_{1}=$ Creation of risk and investment profile. The two steps containing the decision of portfolio development were consolidated to $D_{2}=$ Development of investment proposal. $D_{3}=$ Execution of proposal and $D_{4}=$ Rebalancing complete the initial set of dimensions after this iteration:

$$
T=\left\{\begin{aligned}
D_{1} & =\text { Creation of risk \& investment profile } \\
D_{2} & =\text { Development of investment proposal } \\
D_{3} & =\text { Execution of proposal } \\
D_{4} & =\text { Rebalancing }\}
\end{aligned}\right.
$$

Iteration $1 b$ - Guided by the trade-offs described in chapter 2.2, this iteration is concerned with the derivation of the characteristics $C$ from our theoretical framework. As we integrate the aspects of decision automation and delegation, we distinguish between three levels respectively. Each of the seven resulting combinations depicted in Table 1 make up a

\begin{tabular}{|c|c|}
\hline$D_{i}=\left\{C_{i 1}\right.$ & Self-management \\
\hline$C_{i 2}$ & Human advice \\
\hline$C_{i 3}$ & Hybrid advice \\
\hline$C_{i 4}$ & Algorithmic advice \\
\hline$C_{i 5}$ & Delegation to human \\
\hline$C_{i 6}$ & Delegation to hybrid system \\
\hline$C_{i 7}$ & Delegation to algorithm \} \\
\hline
\end{tabular}
characteristic of our taxonomy. The conceptual grounding resulted in the following preliminary characteristics $C$ :

Iteration 2 - In this iteration, we applied the initial taxonomy to a larger sample of digital investment management systems. In total, we analyzed 62 international providers, selected based on samples from previous literature on robo-advisory [3, 17] and the company database Crunchbase, which focuses on emerging technologies and start-ups. Due to our focus on private investment decisions, 32 providers were excluded from our sample as they either targeted professional asset managers, did not concern investment decisions (e.g. insurance advisors) or were inactive. Our final sample consisted of 30 service offerings from the United States (50\%), Europe (43\%) and Australia (7\%). Inception dates ranged from 2006 to 2017, with three quarters of the sample founded after 2010. For all providers in the final sample, we analyzed the levels of automation and delegation within each of the four process dimensions. For this purpose, two of the co- authors screened provider websites and applied a representative investor profile to the system to document the degrees of automation and delegation applied in the respective process steps. Where relevant information was not attainable following this procedure, we relied on chats and calls with customer support and investment management staff following a pre-defined question outline. Inter-coder reliability was ensured by double-coding a sub-sample of ten systems and a comparison of the assigned characteristics yielding congruence in $91 \%$ of the cases. System attributes that led to the assignment of a certain characteristic within a system dimension were documented, adding to the shared understanding of the coding scheme.

The key finding of our observations in this iteration is that the fund universe available for the development of the investment proposal was typically subject to human pre-selection. Due to the observed difference in automation between the pre-selection of assets and the development of investment proposals, we added the new dimension pre-selection of funds to the taxonomy. In addition, information collected on $D_{3}=$ Execution of proposal implied the irrelevance of execution decisions for current providers. Hence, execution of proposal was discarded from the taxonomy. Thus, we implicitly assume that investors refrain from strategic trading decisions such as market timing, which seems plausible given the contested profitability even for mutual fund managers [37]. In order to maintain the chronological order of the dimensions, pre-selection of funds was coded as $D_{2}$, while the previous $D_{2}=$ Development of investment proposal became $D_{3}$ :

$$
T=\left\{\begin{aligned}
D_{1} & =\text { Creation of risk \& investment profile } \\
D_{2} & =\text { Pre-selection of funds } \\
D_{3} & =\text { Development of investment proposal } \\
D_{4} & =\text { Rebalancing }\}
\end{aligned}\right.
$$

With respect to our set of characteristics, we were able to identify instances of all theoretically derived combinations of automation and delegation within single process steps of the analyzed offerings. Thus, our initial set of characteristics was empirically verified. However, not all characteristics were present within each dimension. Therefore, we restricted the set of characteristics within each dimension to those observed in current systems. As presented in Table 2, there is large variation in the creation of risk \& investment profile $\left(D_{1}\right)$ and rebalancing $\left(D_{4}\right)$ dimensions, each displaying five distinct characteristics. In contrast, the pre-selection of funds $\left(D_{2}\right)$ and development of investment proposal $\left(D_{3}\right)$ dimensions display little variation, each taking on two distinct characteristics. The former $\left(D_{2}\right)$ is fully delegated to the service provider in all cases, whereas the latter $\left(D_{3}\right)$ is conducted exclusively using an algorithm. 
Table 2: Taxonomy after second iteration

\begin{tabular}{|c|c|c|c|c|c|}
\hline & & \multicolumn{4}{|c|}{ Dimensions } \\
\hline \multirow{8}{*}{ 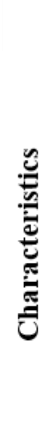 } & & $D_{l}$ & $D_{2}$ & $D_{3}$ & $D_{4}$ \\
\hline & $C_{i I}-$ Self management & $\bullet$ & & & $\bullet$ \\
\hline & $C_{i 2}-$ Human advice & $\bullet$ & & & \\
\hline & $C_{i 3}-$ Hybrid advice & $\bullet$ & & & $\bullet$ \\
\hline & $C_{i 4}$ - Algorithmic advice & $\bullet$ & & $\bullet$ & $\bullet$ \\
\hline & $C_{i 5}$ - Delegation to human & & $\bullet$ & & \\
\hline & $C_{i 6}-$ Delegation to hybrid system & & & & $\bullet$ \\
\hline & $C_{i 7}$ - Delegation to algorithm & $\bullet$ & $\bullet$ & $\bullet$ & • \\
\hline
\end{tabular}

Iteration 3 -Due to the additional dimension and the exclusion of characteristics, the proposed ending conditions have not been met in iteration 2. Thus, we proceeded with data collection in a third iteration to apply the newly adjusted taxonomy. Previously classified systems were re-evaluated based on the newly specified dimensions.

To detect the level of completion of our taxonomy, we were guided by the principle of mutually exclusive and collectively exhaustive dimensions and characteristics and applied the subjective and objective ending conditions suggested by Nickerson et al. [13]. We find the five subjective ending conditions to be met after iteration 3. The taxonomy is concise in terms of a limited number of dimensions and characteristics, robust in terms of differentiation among objects, comprehensive as all objects can be classified, extendible as new dimensions could be added in previous iterations and explanatory due to the provision of understanding of system design gained when applying the taxonomy. The objective ending conditions [13] were met as the entire sample was examined and no object, dimension or characteristic was merged or split in the last iteration and no new dimension or characteristic was added.

Figure 2 illustrates the occurrence of combinations of decision automation and delegation within the four final dimensions as observed in our sample. The size of a bubble indicates the frequency of the respective combination.

The majority of systems fully automate the creation of risk and investment profiles using online questionnaires $\left(D_{l}, 83 \%\right)$, as well as the development of investment proposals $\left(D_{3}, 100 \%\right)$ and rebalancing tasks $\left(D_{4}, 70 \%\right)$. Human conduct is mainly applied in the preselection of funds $\left(D_{2}, 80 \%\right)$, while hybrid solutions are applied mainly in rebalancing $\left(D_{4}, 20 \%\right)$, where suggestions made by an algorithm are reviewed by a human.
The levels of delegation vary substantially between the dimensions. In most systems, users fully delegate decisions regarding the pre-selection of funds $\left(D_{2}\right.$, $100 \%)$, the development of an investment proposal $\left(D_{3}\right.$, $67 \%)$ and rebalancing $\left(D_{4}, 63 \%\right)$. Partial delegation is typically applied in the creation of risk profiles $\left(D_{l}\right.$, $67 \%$ ), implying that users are often able to adjust the risk profile proposed by the system. In some instances, the creation of risk profiles $\left(D_{l}, 7 \%\right)$ and rebalancing $\left(D_{4}, 10 \%\right)$ are not delegated since they are not offered as part of the service.

\subsection{Discussion of taxonomy}

Overall, the data we collected during taxonomy development provide an answer to the question as to what levels of decision automation and delegation are applied by current digital investment management systems. In the creation of risk \& investment profile dimension $\left(D_{l}\right)$, the majority of systems apply a questionnaire containing a number of questions regarding the user's financial circumstances and risk preferences. To translate the information provided by the user into a risk profile, more than $80 \%$ of providers use an algorithm. Risk profiling practices are subject to criticism as they have been shown to oversimplify and only make use of less than two thirds of the questions asked to produce the profile [21]. In the scope of a concise questionnaire, this difficulty of creating a comprehensive user profile remains. Therefore, the restriction of information from the risk questionnaire limits the quality of the risk profile produced by the algorithm. This flawed profile then acts as an input to further algorithmic processing in succeeding steps potentially leading to decreases in the quality of the final system output. The fact that most systems $(67 \%$, see Figure 2) partially refrain from autonomy in creating a final risk profile presumably reflects the attempt to avoid severe misclassifications while still maintaining a beneficial level of autonomy for the majority of users.

Some providers warn users when they attempt to manually adjust the proposed profile or limit the scope of possible adjustments, only facilitating users whose profiles have been severely misclassified to decrease the scope of decision delegation by conducting manual changes. Observations in this dimension distinctly point at the inherent system design trade-off between allowing user control while ensuring the realization of benefits from autonomy.

The pre-selection of funds $\left(D_{2}\right)$ is conducted by humans in $80 \%$ of the observed systems, implying that the pool of funds available for selection into a portfolio is typically determined by an investment team. This 

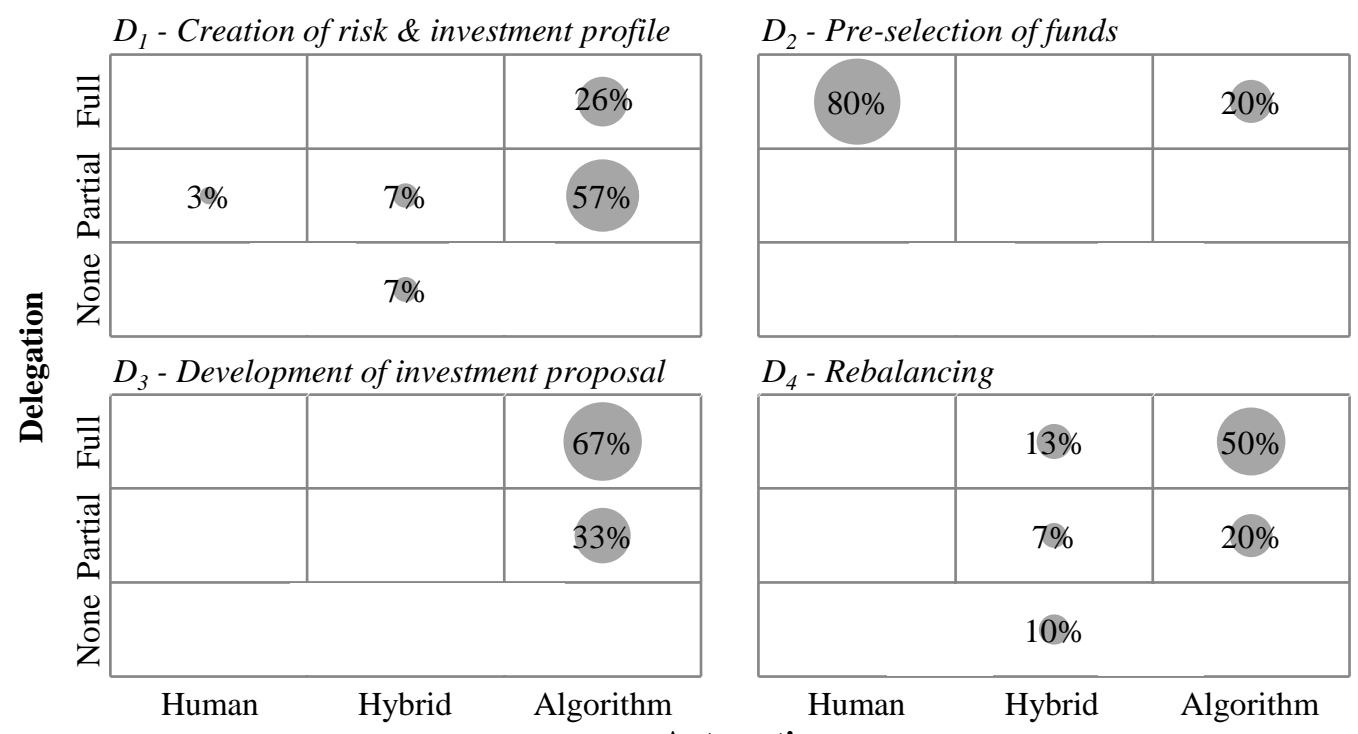

Automation

Human Hybrid Algorithm

Figure 2: Frequency of observed combinations of automation and delegation by dimension

restriction of the algorithm's choice set in the succeeding process step potentially prevents full realization of performance advantages from the automation of portfolio allocation $\left(D_{3}\right)$. The severe impact of this restriction for the final output of the system implies that the determination of the benefits of digital investment management systems requires studying both the aggregate level of automation, as well as the steps in which automation does or does not occur. In addition to the failure to realize the full potential of performance improvements from algorithmic conduct, human input selection might bias the portfolio composition towards assets of affiliated providers [23]. At the same time, users lack control over the preselection of assets in the entire sample, which prevents them from mitigating potential conflicts of interest.

The development of investment proposal $\left(D_{3}\right)$ is concerned with determining suitable portfolio weights based on the risk profile $\left(D_{1}\right)$ and the available fund universe $\left(D_{2}\right)$. Decisions in this dimension are conducted algorithmically in all and fully autonomously in $67 \%$ of systems. The particularly high level of automation in this step characterizes digital investment management and builds the foundation of the propagated value proposals. Due to the centrality to output creation and the high visibility, users might primarily base their adoption of the entire system on the decision automation and delegation in this dimension $\left(D_{3}\right)$.

Within the rebalancing dimension $\left(D_{4}\right)$, the constant monitoring of changes in portfolio composition due to market movements and (suggested) realignment with the risk profile is conducted algorithmically in $70 \%$ of the cases. This coincides with the ability of algorithmic systems to constantly process large numbers of observations and react immediately if required. It stands to reason that the high level of delegation in rebalancing (full delegation in $63 \%$ of systems, see Figure 2) reflects providers' confidence in the suitability of algorithmic conduct for this task as well as users' appreciation of decreased effort from ongoing portfolio monitoring.

\section{Archetypes of digital investment management systems}

The taxonomy developed in the previous chapter consists of four dimensions and a total of seven potential characteristics. Thus, in theory, 2,401 unique combinations of decision automation and delegation are possible, of which 100 can be assumed practically viable (see Table 2). Due to this vast number of possible combinations, identifying archetypes with regards to dimensions and characteristics is necessary to reduce complexity and identify frequent and distinct patterns, posing new questions for system design. In addition, findings from the taxonomy, such as the prevalence of human restriction of algorithmic input have illustrated the importance of an aggregated view of automation and delegation along the investment management process.

\subsection{Derivation of archetypes}

Based on our taxonomy, we developed archetypes of digital investment management systems in a two-step procedure. In the first step, we identified archetypes separately for the aspects of delegation and automation. The second step consolidated the two dimensions and 
proposed integrated archetypical systems. To cluster existing system designs, we first identified designs that were either distinct or occurred frequently. We then consolidated systems displaying only minor differences in their characteristics [38]. This consolidation was achieved by allocating a system to the cluster to which it added the least variance with respect to characteristics. To ensure robustness and conciseness, we then calculated and compared the resulting within- and between-archetype variances of characteristics. Withinvariance was consistently lower than between-variance, which indicated that clusters were explanatory in the sense that the included systems were similar, and parsimonious in the sense that systems from different archetypes were sufficiently distinct.

Delegation archetypes - Figure 3 displays the four identified archetypes differentiated by levels of decision delegation. Due to the consolidation of related systems based on the similarity of their characteristics, different delegation levels occurred within a single step of an archetype. We indicated these combinations with quarters (none or partial) and three-quarters (partial or full) in Figure 3.

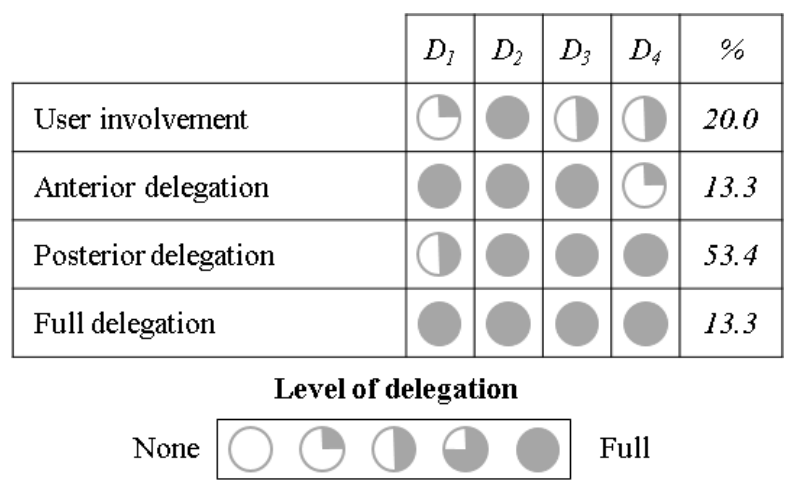

Figure 3: Archetypes according to level of delegation

As previously discussed, all systems in our sample are delegated full decision authority with respect to the pre-selection of assets eligible for inclusion in the portfolios $\left(D_{2}\right)$. Thus, our clustering with respect to decision delegation is based on the characteristics of the remaining three dimensions. The user involvement archetype, which applies to $20 \%$ of systems, is characterized by comprehensive user control. Users are able to adjust the proposed risk profile $\left(D_{l}\right)$ and assigned portfolio weights $\left(D_{3}\right)$ as well as implement rebalancing decisions $\left(D_{4}\right)$. Anterior delegation refers to systems that create a risk profile $\left(D_{1}\right)$ and assemble a matching investment proposal $\left(D_{3}\right)$ without any user involvement. Rebalancing decisions $\left(D_{4}\right)$ proposed by the system have to be confirmed by the user, implying only partial delegation toward the end of the process. Posterior delegation systems apply only partial delegation in the front of the process, allowing users to adjust the proposed risk profile $\left(D_{1}\right)$. Based on this profile, the system develops $\left(D_{3}\right)$ and maintains $\left(D_{4}\right)$ a suitable portfolio autonomously. Posterior delegation was observed in more than half of the services. Full delegation systems conduct decisions fully autonomously along the entire investment management process $\left(D_{1}-D_{4}\right)$, solidifying the evolution of services from portfolio advice to delegated investment management.

Automation archetypes - As displayed in Figure 4, we identified three unique system archetypes with respect to the level of automation. In analogy to Figure 3 , dimensions consolidating multiple automation levels are indicated as shaded. The first generation of systems provides at least partially automated risk profiling $\left(D_{l}\right)$ which is utilized in a fully automated portfolio allocation $\left(D_{3}\right)$. The assets eligible for portfolio allocation are exclusively selected by humans $\left(D_{2}\right)$, while implementation of trades and rebalancing $\left(D_{4}\right)$ are not part of the service. Due to the centrality of automated investment proposal development, we refer to this archetype as portfolio allocation. As discussed in the previous chapter, the majority of systems (70\%) are characterized by a partially or fully automated creation of risk profiles $\left(D_{2}\right)$ that are algorithmically translated into a portfolio $\left(D_{3}\right)$ based on assets selected by humans $\left(D_{2}\right)$. Rebalancing $\left(D_{4}\right)$ is at least partially automated. Due to the constrained input of the algorithmic proposal development, we refer to this archetype as bounded automation. Finally, 20\% of providers consistently follow an algorithmic approach to decision-making in all process steps. We call this type full automation.

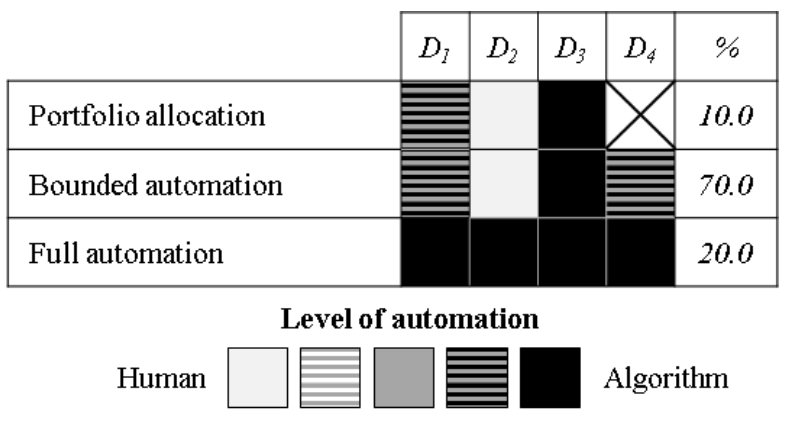

Figure 4: Archetypes according to level of automation

Integrated archetypes - To account for interdependencies between the levels of decision automation and delegation, we unify the two aspects and propose five integrated system archetypes. To illustrate the types in a comprehensible manner without oversimplifying, Figure 5 combines the graphical 
representation of delegation levels as suggested in Figure 3 and automation levels as suggested in Figure 4. As in the separate development of automation and delegation archetypes, similar systems are consolidated such that the within-archetype variance is minimized. The portfolio allocator archetype - making up $10 \%$ of the systems - is adopted from the delegation clustering. It is characterized by a lack of implementation and rebalancing of proposed portfolios $\left(D_{4}\right)$, as well as fully automated and delegated portfolio allocation $\left(D_{3}\right)$. Investment advisors allow the user to adjust the algorithmically proposed risk profiles $\left(D_{l}\right)$ and portfolio compositions $\left(D_{3}\right)$ and require the confirmation of rebalancing decisions $\left(D_{4}\right)$. Automation is constrained by the fully autonomous human pre-selection of assets $\left(D_{2}\right)$. Guided delegate systems exhibit full automation and delegation toward the end of the process, which refers to portfolio weight allocation $\left(D_{3}\right)$ and rebalancing decisions $\left(D_{4}\right)$. The risk profile $\left(D_{1}\right)$ serving as an input to these posterior steps is guided by the user. This archetype is observed most frequently in the data, making up $53 \%$ of systems. Finally, boundedautomation delegate and full-automation delegate systems do not require the user to participate in decision-making in any process step $\left(D_{1}-D_{4}\right)$. They mainly differ in the level of automation in the preselection of assets $\left(D_{2}\right)$.

Systems previously assigned the anterior delegation archetype do not constitute a distinct integrated archetype due to the scarcity of their occurrence and their similarity with other integrated archetypes. Based on the variance analysis these systems were assigned to the integrated archetypes portfolio allocator and bounded-automation delegate.

\begin{tabular}{|c|c|c|c|c|c|}
\hline & $D_{l}$ & $D_{2}$ & $D_{3}$ & $D_{4}$ & $\%$ \\
\hline Portfolio allocator & $\Leftrightarrow$ & & & & 10.0 \\
\hline Investment advisor & & & & & 16.7 \\
\hline Guided delegate & & & & & 53.3 \\
\hline Bounded-automation delegate & & & & & 16.7 \\
\hline Full-automation delegate & & & & & 3.3 \\
\hline
\end{tabular}

\section{Automation}

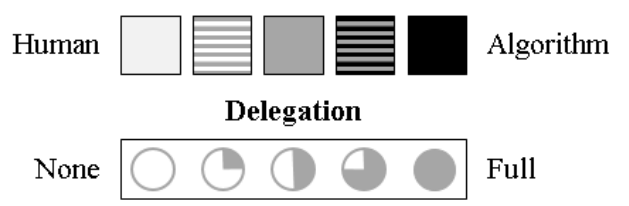

Figure 5: Integrated system archetypes

\subsection{Discussion of archetypes}

From the automation archetypes derived above, we observe that current systems are highly automated with respect to the creation of risk profiles $\left(D_{l}\right)$, as well as the development of investment proposals $\left(D_{3}\right)$ and rebalancing $\left(D_{4}\right)$. In contrast, providers frequently choose not to automate the pre-selection of assets $\left(D_{2}\right)$, which limits the effectiveness of digital investment management systems due to human restriction of the choice set for algorithms in subsequent steps $\left(D_{3}\right.$ and $D_{4}$ ). In addition, this lack of automation in fully delegated decisions may increase user suspicions of conflicts of interest [22, 23].

From the delegation archetypes, we learn that digital investment management has evolved from portfolio advisory to delegated investment management. Thus, the term robo-advisor, which was originally created for systems that provided portfolio allocation recommendations based on risk questionnaires without implementing these recommendations, may no longer be an accurate description of all current providers. This is why we refer to the new generation of robo-advisors (anterior, posterior and full delegation) as digital investment management systems, reflecting the increased level of delegation.

Further, decision authority is frequently delegated to the systems toward the end of the process, while prior decisions such as the creation of a risk profile $\left(D_{l}\right)$ are often subject to user participation. This is due to the difficulty of creating an accurate risk profile based on parsimonious questionnaires [21]. Choosing to involve the user in this dimension may reflect a lack of confidence in the quality of the risk profile, as well as an attempt to compensate the user by partially increasing control.

The development of integrated archetypes revealed that a majority of systems rely on partially delegated and highly automated risk profiling $\left(D_{1}\right)$, autonomous human pre-selection of assets $\left(D_{2}\right)$ and fully autonomous and automated portfolio development $\left(D_{3}\right)$ and rebalancing $\left(D_{4}\right)$. These systems implement favorable levels of delegation by taking over the decisions that require the most immediate and recurring actions $\left(D_{4}\right)$. To identify the necessity of these actions, providers mostly refer to algorithms to constantly monitor market and portfolio developments, decreasing human effort.

It is important to note that neither the extent of automation, nor the level of delegation separately determine the quality of an investment management system. In the risk profiling dimension $\left(D_{l}\right)$, highly automated decision-making has been shown to yield inaccurate profiles, which can be mitigated by decreasing the level of delegation to the system by 
involving the user. In the pre-selection dimension $\left(D_{2}\right)$, the lack of user involvement facilitates conflicts of interest, which can be offset by applying an algorithm, thus increasing the level of automation. The prevalence of algorithmic conduct in the risk profiling dimension $\left(D_{1}\right)$, as well as full delegation in the pre-selection dimension $\left(D_{2}\right)$ implies that few providers have addressed these issues. Therefore, we conclude that both benefits and issues regarding an investment management system arise from certain combinations of decision automation and delegation.

\section{Conclusion and future research}

In this study, we investigated the degrees of customer involvement and automation in the decisionmaking along the investment management process. Due to the impact of the levels of automation (algorithm aversion) and delegation (user control) on the adoption of algorithmic systems, these two aspects lend themselves as suitable differentiators of such systems. The dimensions proposed in the taxonomy identify decisions that can be delegated to and automated by a digital service provider. The characteristics provide an integrated measure for the degree of delegation and automation for each dimension (i.e. decision).

The developed taxonomy allowed us to derive archetypical systems representing prevalent types of service providers and their characteristics. These archetypes facilitate further research on the perception and adoption of digital service providers in the investment management domain.

Based on the taxonomy and the derived archetypes, we find that decisions requiring constant attention and immediate action such as rebalancing $\left(D_{4}\right)$ tend to be highly automated and delegated, resulting in reduced user effort. Process steps in which algorithmic decisionmaking is inaccurate such as in risk profiling $\left(D_{1}\right)$ tend to be only partially delegated to mitigate potential errors. Interestingly, central parts of the process such as the pre-selection of funds $\left(D_{2}\right)$ are not automated, limiting the efficiency gains from service automation.

The development of our taxonomy draws on a set of implicit and explicit assumptions and discretionary judgement, which should be kept in mind when applying it. In particular, the final selection of the relevant dimensions may have been subject to the researchers' preconceptions. We have addressed this concern by combining information from multiple sources. To avoid subjectivity in data collection, we rely on double-coding for the classification of decision-making within the process steps.

Based on the insights from this study, we propose that future studies should integrate automation and delegation to identify the unbiased drivers of system adoption. Separate analyses might be subject to omitted variable biases if decision automation and delegation were correlated. Thus, only an integrated evaluation would allow for the identification of the optimal system design aligning the two aspects.

\section{References}

[1] Eickhoff, M., Muntermann, J., and Weinrich, T., "What Do FinTechs Actually Do? A Taxonomy of FinTech Business Models", International Conference on Information Systems, 2017.

[2] Gozman, D., Liebenau, J., and Mangan, J., "The Innovation Mechanisms of Fintech Start-Ups: Insights from SWIFT's Innotribe Competition", Journal of Management Information Systems, 35(1), 2018, pp. 145-179.

[3] Jung, D., Dorner, V., Glaser, F., and Morana, S., "RoboAdvisory", Business \& Information Systems Engineering, 60(1), 2018, pp. 81-86.

[4] Sironi, P., FinTech Innovation: From Robo-advisors to Goal Based Investing and Gamification, Wiley \& Sons, Cornwall (USA), 2016.

[5] Harvey, C.R., Rattray, S., Sinclair, A., and Van Hemert, O., "Man vs. Machine: Comparing Discretionary and Systematic Hedge Fund Performance", The Journal of Portfolio Management, 43(4), 2017, pp. 55-69.

[6] Dawes, R.M., Faust, D., and Meehl, P.E., "Clinical versus Actuarial Judgment", Science, 243(4899), 1989, pp. 16681674.

[7] D'acunto, F., Prabhala, N., and Rossi, A., "The Promises and Pitfalls of Robo-advising", Review of Financial Studies (cond. acc.), 2018.

[8] Grove, W.M., Zald, D.H., Lebow, B.S., Snitz, B.E., and Nelson, C., "Clinical Versus Mechanical Prediction: A Metaanalysis", Psychological Assessment, 12(1), 2000, pp. 19.

[9] Barkhuus, L., and Dey, A., "Is Context-aware Computing Taking Control Away from the User? Three Levels of Interactivity Examined", International Conference on Ubiquitous Computing, 2003, pp. 149-156.

[10] Van Der Heijden, H., "Ubiquitous Computing, User Control, and User Performance: Conceptual Model and Preliminary Experimental Design", Research Symposium on Emerging Electronic Markets, 2003, pp. 107-112.

[11] Dietvorst, B.J., Simmons, J.P., and Massey, C., "Algorithm Aversion: People Erroneously Avoid Algorithms After Seeing Them Err", Journal of Experimental Psychology: General, 144(1), 2015, pp. 114. 
[12] Dietvorst, B.J., Simmons, J.P., and Massey, C., "Overcoming Algorithm Aversion: People Will Use Imperfect Algorithms If They Can (Even Slightly) Modify Them", Management Science, 64(3), 2018, pp. 1155-1170.

[13] Nickerson, R.C., Varshney, U., and Muntermann, J., "A Method for Taxonomy Development and its Application in Information Systems", European Journal of Information Systems, 22(3), 2013, pp. 336-359.

[14] Agarwal, S., Chomsisengphet, S., and Lim, C., "What Shapes Consumer Choice and Financial Products? A Review", Annual Review of Financial Economics, 9(1), 2017, pp. 127146.

[15] Collins, J.M., "Financial Advice: A Substitute for Financial Literacy?", Financial Services Review, 21(4), 2012,

[16] Mullainathan, S., Noeth, M., and Schoar, A., "The Market for Financial Advice: An Audit Study", 2012.

[17] Nueesch, R., Zerndt, T., Alt, R., and Ferretti, R.G., "Tablets Penetrate the Customer Advisory Process: A Case from a Swiss Private Bank", Bled eConference, 2016, pp. 202305.

[18] Cocca, T., "Potential and Limitations of Virtual Advice in Wealth Management", Journal of Financial Transformation, 44(1), 2016, pp. 45-57.

[19] Hodge, F.D., Mendoza, K.I., and Sinha, R.K., "The Effect of Humanizing Robo-Advisors on Investor Judgments", 2018.

[20] Reher, M., and Sun, C., "Robo Advisers and Mutual Fund Stickiness", 2016.

[21] Tertilt, M., and Scholz, P., "To Advice, or Not to Advice - How Robo-advisors Evaluate the Risk Preferences of Private Investors", 8th World Finance Conference, 2017.

[22] Fein, M.L., "Robo-advisors: A Closer Look", Banking \& Insurance eJournal, 7(174), 2015.

[23] Ji, M., "Are Robots Good Fiduciaries? Regulating Roboadvisors under the Investment Advisers Act of 1940", Columbia Law Review, 2017, pp. 1543-1583.

[24] Eastwood, J., Snook, B., and Luther, K., "What People Want from Their Professionals: Attitudes toward Decisionmaking Strategies", Journal of Behavioral Decision Making, 25(5), 2012, pp. 458-468.

[25] Diab, D.L., Pui, S.Y., Yankelevich, M., and Highhouse, S., "Lay Perceptions of Selection Decision Aids in US and Non-US Samples", International Journal of Selection and Assessment, 19(2), 2011, pp. 209-216.
[26] Highhouse, S., "Stubborn Reliance on Intuition and Subjectivity in Employee Selection", Industrial and Organizational Psychology, 1(3), 2008, pp. 333-342.

[27] Promberger, M., and Baron, J., "Do Patients Trust Computers?", Journal of Behavioral Decision Making, 19(5), 2006, pp. 455-468.

[28] Önkal, D., Goodwin, P., Thomson, M., Gönül, S., and Pollock, A., "The Relative Influence of Advice from Human Experts and Statistical Methods on Forecast Adjustments", Journal of Behavioral Decision Making, 22(4), 2009, pp. 390409.

[29] Rijsdijk, S.A., and Hultink, E.J., "'Honey, Have You Seen Our Hamster?" Consumer Evaluations of Autonomous Domestic Products", Journal of Product Innovation Management, 20(3), 2003, pp. 204-216.

[30] Ajzen, I., "The Theory of Planned Behavior", Organizational Behavior and Human Decision Processes, 50(2), 1991, pp. 179-211.

[31] Onnasch, L., Wickens, C.D., Li, H., and Manzey, D., "Human Performance Consequences of Stages and Levels of Automation", Human Factors, 56(3), 2014, pp. 476-488.

[32] Parasuraman, R., Sheridan, T.B., and Wickens, C.D., "A Model for Types and Levels of Human Interaction with Automation", IEEE Transactions on SMC, 30(3), 2000, pp. 286-297.

[33] Williams, K., Chatterjee, S., and Rossi, M., "Design of Emerging Digital Services: A Taxonomy", European Journal of Information Systems, 17(5), 2008, pp. 505-517.

[34] Rizk, A., Bergvall-Kåreborn, B., and Elragal, A., "Towards a Taxonomy for Data-Driven Digital Services", Proceedings of the 51st Hawaii International Conference on System Sciences, 2018, pp. 1076-1085.

[35] Financial Planning Standards Board, "Financial Planning in Six Steps", (04/10/2018) retrieved from https://www.fpsb.org

[36] Bhattacharya, U., Hackethal, A., Kaesler, S., Loos, B., and Meyer, S., "Is Unbiased Financial Advice to Retail Investors Sufficient?", Review of Financial Studies, 25(4), 2012, pp. 975-1032.

[37] Cuthbertson, K., Nitzsche, D., and O'Sullivan, N., "The Market Timing Ability of UK Mutual Funds", Journal of Business Finance \& Accounting, 37(1-2), 2010, pp. 270-289.

[38] Bailey, K.D., Typologies and Taxonomies: An Introduction to Classification Techniques, Sage, Thousand Oaks (USA), 1994. 